\title{
Defective INPP5E distribution In NPHP1-related Senior-Løken Syndrome
}

\author{
Ke Ning ${ }^{1}$, Emilie Song ${ }^{1}$, Brent Sendayen ${ }^{1}$, Philipp Prosseda ${ }^{1}$, Kun-Che Chang ${ }^{1}$, Alireza \\ Ghaffarieh $^{2}$, Jorge Alvarado ${ }^{1}$, Biao Wang ${ }^{1}$, Nicolas Berbari ${ }^{3}$, Yang $\mathrm{Hu}^{1}$, and Yang Sun ${ }^{1}$ \\ ${ }^{1}$ Stanford University School of Medicine \\ ${ }^{2}$ Harvard Medical School Department of Medicine \\ ${ }^{3}$ Indiana University Purdue University at Indianapolis
}

August 23, 2020

\begin{abstract}
Senior-Løken syndrome is a rare genetic disorder which presents with nephronophthisis and retinal degeneration, leading to end-stage renal disease and progressive blindness. The most frequent cause of juvenile nephronophthisis is mutation in the nephronophthisis type 1 (NPHP1) gene. NPHP1 encodes the protein nephrocystin-1, which functions at the transition zone (TZ) of primary cilia. Here we report a 9-year-old Senior-Løken syndrome patient with NPHP1 deletion, who presents with a decreased electroretinogram consistent with early retinal degeneration. The patient had undergone bilateral nephrectomy and a renal transplant. Immunohistochemistry and electron microscopy of the resected kidney showed disorganized cystic structures with loss of cilia in renal tubules. Phosphoinositides have been recently recognized as critical components of the ciliary membrane and immunostaining of kidney sections for phosphoinositide 5-phosphatases (INPP5E) showed loss of staining compared to a healthy control. The decreased expression of INPP5E specifically in the primary cilium, coupled with disorganized cilia morphology, suggests a novel role of NPHP1 that it is involved in regulating ciliary phosphoinositide in the ciliary membrane of renal tubular cells.
\end{abstract}

\begin{abstract}
Senior-Løken syndrome is a rare genetic disorder which presents with nephronophthisis and retinal degeneration, leading to end-stage renal disease and progressive blindness. The most frequent cause of juvenile nephronophthisis is mutation in the nephronophthisis type 1 (NPHP1) gene. NPHP1 encodes the protein nephrocystin-1, which functions at the transition zone (TZ) of primary cilia. Here we report a 9-year-old Senior-Løken syndrome patient with NPHP1 deletion, who presents with a decreased electroretinogram consistent with early retinal degeneration. The patient had undergone bilateral nephrectomy and a renal transplant. Immunohistochemistry and electron microscopy of the resected kidney showed disorganized cystic structures with loss of cilia in renal tubules. Phosphoinositides have been recently recognized as critical components of the ciliary membrane and immunostaining of kidney sections for phosphoinositide 5-phosphatases (INPP5E) showed loss of staining compared to a healthy control. The decreased expression of INPP5E specifically in the primary cilium, coupled with disorganized cilia morphology, suggests a novel role of NPHP1 that it is involved in regulating ciliary phosphoinositide in the ciliary membrane of renal tubular cells.
\end{abstract}

Key Words: Primary cilia, NPHP1, Senior-Løken syndrome, nephronophthisis, transition zone, INPP5E

The primary cilium is a solitary, immotile microtubule-based structure extending from the surface of almost all mammalian cell types (Anderson et al., 2008; Berbari, O'Connor, Haycraft, \& Yoder, 2009; Satir \& Christensen, 2007; Veland, Awan, Pedersen, Yoder, \& Christensen, 2009). Defects in cilia result in a class 
of multi-organ diseases that usually include retinal degeneration, renal disease and cerebral abnormalities collectively called ciliopathies (Halbritter et al., 2013; Hildebrandt, Attanasio, \& Otto, 2009). Nephronophthisis (NPHP) is an autosomal recessive cystic renal ciliopathy characterized in the clinic by polyuria and anemia as core manifestations. NPHP constitutes the most frequent genetic cause of end-stage kidney disease before age 30 (Ahmed \& Ali, 2011; Srivastava \& Sayer, 2014; Stokman, Lilien, \& Knoers, 1993). Deletion of NPHP1, an important ciliary protein residing at the ciliary transition zone (TZ), is associated with development of Senior-Løken syndrome and NPHP, which mainly affects the kidneys and eyes (Ronquillo, Bernstein, \& Baehr, 2012).

In addition to retinal degeneration and kidney defects, clinical manifestations of Senior-Løken syndrome may also include skeletal, dermatological and cerebellar anomalies, small hand (short metacarpals) and madarosis (Aggarwal, Jain, Yadav, Kaverappa, \& Gupta, 2013; Ronquillo et al., 2012). Currently, no treatment is available to prevent disease pathogenesis. Deletion in the NPHP1 gene represents the leading genetic abnormality reported in nephrophthisis patients to date (Hildebrandt et al., 2009; Wolf, 2015). Similar to other NPHP gene product, the NPHP1 protein has been found to localize at the basal body and the TZ of primary cilia in renal tubular cells and retinal photoreceptors (Fliegauf et al., 2006; Garcia-Gonzalo \& Reiter, 2017; Gogendeau et al., 2020; Goncalves \& Pelletier, 2017; Omran, 2010). NPHP1 has also been found in areas of cell to cell contact, including tight junctions, adherens junctions and focal adhesions (Hildebrandt et al., 2009; Zhou et al., 2012).

The ocular involvement in Senior-Løken syndrome is variable and includes different degrees of retinal dystrophy and cataracts (Aggarwal et al., 2013). The most frequently reported ophthalmologic manifestations of NPHP have been retinal degeneration and ocular-motor apraxia (OMA) (Aggarwal et al., 2013; R, 2014). In this study, we report the clinical phenotype of a 9-year-old male patient. The child initially presented with progressive renal failure at age 2. Subsequent ultrasound findings were consistent with cystic kidney disease. He went on to develope renal failure and early signs of retinal degeneration. The patient presented with polyuria and polydipsia, then progressed to end-stage renal disease, for which he underwent bilateral nephrectomy and a renal transplant. Ophthalmic exam showed his visual acuity was 20/20 in both eyes. On slit lamp examination, the anterior segment of the both eyes was also normal. Color fundus photographs of both eyes were unremarkable, without pigmentary changes, lesions or scars in the macula or periphery (Fig 1A ). Visual field examination showed no focal defects in both eyes (Fig 1B ). Color fundus photographs in NPHP1 patients are typically normal despite bilateral visual field loss. Full field ERG, which provides an assessment of overall retinal function, was recorded after 30 minutes of dark adaptation. The scotopic and photopic flash ERG showed a decreased rod and cone response in both eyes (Fig 1C). The amplitude of the b-wave was reduced for the rod response (0.01, 3.0 and 10.0 scotopic) without implicit time delay. The b value of the dark-adapted ERG (0.01) was $36 \mu \mathrm{V}$ OD compared with $140 \mu \mathrm{V}$ in the control patient. ERG (3.0) was $120 \mu \mathrm{V}$ OD compared with $212 \mu \mathrm{V}$ in the control patient. ERG (10.0) was $152 \mu \mathrm{V}$ OD compared with $217 \mu \mathrm{V}$ in the control patient. The amplitude of both a and b-wave of the cone response was bilaterally reduced compared to the age-matched control. The $\mathrm{b}$ value of the light-adapted ERG was $50 \mu \mathrm{V}$ OD in the patient and $143 \mu \mathrm{V}$ in the control patient. No remarkable changes were found in $30-\mathrm{Hz}$ flicker in either eye (data not shown). In summary, the patient with NPHP1 deletion demonstrated diminished rod responses and overall impaired cone function.

Kidneys of NPHP1 patients display a characteristic triad of corticomedullary cysts, tubular basement membrane disruption and tubulointerstitial nephropathy (Srivastava \& Sayer, 2014; Stokman, Lilien, \& Knoers, 1993; Wolf, 2015). To determine the renal phenotype of this patient, histopathological analysis of his kidney obtained by nephrectomy was performed alongside an identical analysis of a healthy human kidney (Fig. 1D ). H\&E staining of the patient's kidney sections showed a diffuse sclerosing tubulointerstitial process with a predominance of tortuous and atrophic tubules at the corticomedullary junction. Enlarged cortical cyst formation was present in the corticomedullary region. Higher magnification highlighted a thickened and multilayered tubular basement membrane (TBM). In order to determine the impact of mutation of NPHP1 on human primary cilia and TBM of renal epithelia cells, and TBM profiles by transmission electron microscopy. Electron microscopy revealed an irregular TBM pattern, consisting frequently of two or three 
membrane layers which were folded excessively while the lining of the tubular cysts was flattened. As shown in Figure 1D, fewer primary cilia were seen on the apical side of the tubular epithelial cells, indicating renal cilia dysfunction.

It has been previously demonstrated that nephrocystin proteins localize to the ciliary TZ of renal tubular epithelia in mice and cultured cells. The TZ, a cilia sub-compartment just distal of the basal body, has a unique membrane content and has also been implicated in regulating what proteins enter the cilia signaling compartment in diverse model systems (Awata et al., 2014; Fliegauf et al., 2006; Sang et al., 2011; Shi et al., 2017; Szymanska \& Johnson, 2012; Williams et al., 2011). To our knowledge (Fliegauf et al., 2006) cilia frequency, morphology and content of renal primary cilia in a human patient with NPHP1 deletion has not been done. Here, we assess primary cilia in a kidney biopsy of a patient with nephronophthisis. We examined the renal biopsy with confocal immunofluorescence microscopy and compared the findings to those in relevant control samples from a healthy kidney. Typical ciliary markers, acetylated $\alpha$-tubulin an axoneme marker, Arl13b a cilia membrane marker and Intraflagellar Transport 88 a cilia specific IFT marker, were employed to assess primary cilia. Compared with control kidney primary cilia, NPHP1 patient renal cilia were disorganized and shortened (Fig. 2A ). Quantification of cilia which are marked by Arl13b antibody frequency showed loss of NPHP1 significantly reduced the proportion of ciliated cells from $78.5 \pm 12.3 \%$ in the normal patient to $60 \pm 7.3 \%$ in the NPHP1 deletion patient, indicating that NPHP1 influences ciliogenesis in human renal tubular cells (Fig. 2E ). In addition, quantification of immunofluorescently labeled primary cilia revealed that ciliary length was reduced from $5.6 \pm 1.2 \mu \mathrm{m}$ in the healthy patient to $3.2 \pm 1.0 \mu \mathrm{m}$ in the NPHP1 patient (Fig. 2F ). Previous studies have shown that mice with NPHP1 deletion do not develop pathological characteristics of nephronophthisis (Jiang et al., 2008). However, NPHP1 knockout significantly decreases ciliation and ciliary length in MDCK cells, which is consistent with our data for patient-derived cilia (Delous et al., 2009). This difference in phenotype might be due to species differences in gene function.(Williams, Masyukova, \& Yoder, 2010). Recent data have also begun to demonstrate that TZ function may be cell type and tissue specific, with mutations in some TZ members having specific phenotypes (Lewis et al., 2019). Our findings suggest that NPHP1 may be required for normal cilia maintenance and function in human kidney.

The TZ is a compartment of the proximal region of the cilium, between the basal body and axoneme. Numerous studies in diverse model systems have shown that TZ functions as a selective membrane diffusion barrier, regulating ciliary protein entry and exit (Goncalves \& Pelletier, 2017; Omran, 2010). Two TZ protein complexes have been identified: the NPHP complex and MKS complex. In mammalian cells, AC3 and PKD2 (adenylyl cyclase III and PKD2 are both ciliary proteins) are aberrantly located in MKS-complexdeficient cilia (Garcia-Gonzalo et al., 2011). InC.elegans , TRAM protein and membrane-associated RP2 homologues are abnormal in TZ-deficient cilia (Williams et al., 2011). Previous studies have demonstrated by LAP-tagging and in vitro binding, that NPHP1, NPHP4 and NPHP8 have a strong mutual interaction (Sang et al., 2011): NPHP4 acts as a bridge that directly binds both NPHP1 and NPHP8in vitro, whereas NPHP1 and NPHP8 cannot directly bind to each other. Furthermore, NPHP4 and NPHP8 have been shown to localize to the base of the cilia of renal tubular cells in vivo and in vitro (Delous et al., 2009; Sang et al., 2011), evidence that they play a clear role within cilia. Based on these studies, we hypothesized that loss of NPHP1 would directly affect NPHP4 localization in vivo, but produce no alteration of NPHP8. To test this hypothesis, we co-stained renal sections from the patient with NPHP1 deletion and healthy controls for either NPHP4 or NPHP8 and the ciliary marker Arl13b. Analysis of confocal images revealed no alteration in NPHP8 localization, indicating that NPHP1 exerts its function independently of NPHP8 (Fig 2B ). In healthy control, NPHP4 localized only at the base of the cilia. In contrast, in renal sections from the patient with NPHP1 deletion, NPHP4 was significantly increased not only at the base of the cilia but also in the ciliary axoneme (Fig. 2C ). Quantitative analysis showed that $97.78 \pm 3.85 \%$ renal epithelial cells in the NPHP1 deletion group demonstrated an abnormal enrichment of NPHP4, compared to only $4.2 \pm 7.2 \%$ in the control group (Fig. 2G ). These results suggest that the NPHP1-NPHP4 interaction is conserved and that it plays a role in controlling NPHP4 localization in primary cilia of human renal epithelial cells.

NPHP proteins are important regulators of ciliary entrance and exit for a diverse array of components (Omran, 2010). Therefore, the regulation of phosphoinositides, which recently have been recognized as a 
critical component of the ciliary membrane, could also be influenced by these complexes residing in the ciliary transition zone. Several groups have shown that a mutation of inositol 5-phosphatase (INPP5E), which is found to localize in the axoneme of the primary cilium, is responsible for ciliary instability in Joubert syndrome (Chavez et al., 2015; Dyson et al., 2017; Jacoby et al., 2009). Based on the previously described distribution of NPHP1 in cilia, we hypothesized that NPHP1 may be critical for controlling phosphoinositide entry into the cilia of human renal epithelial cells. Using a monoclonal antibody against INPP5E, we assessed the distribution of INPP5E within the primary cilia of renal tissue from both a patient with NPHP1 deletion and a healthy control. While INPP5E was expressed along the axoneme of primary cilia in normal tissue, it was absent from the cilia of the NPHP1 deletion patient (Fig. 2D ). Quantitative analysis showed that up to $42.40 \pm 13.83 \%$ of renal epithelial cells in the NPHP1 deletion patient had abnormal INPP5E expression, compared to healthy tissue (Fig. 2H ). This data indicates a critical role for NPHP1 in phosphoinositide regulation.

Interestingly, nephronophthisis and Joubert syndrome are two types of ciliopathies with overlapping clinical manifestations, including cystic kidney disease and retinal degeneration. Previous publications showed that NPHP1 and INPP5E mutations are responsible for Joubert syndrome(Constable, Long, Floyd, Schurmans, \& Caspary, 2020; Jacoby et al., 2009). Our study shows that NPHP1 deletion results in abnormal expression of INPP5E, which supports the hypothesis that Joubert syndrome and nephronophthisis represent the mild and the severe versions of the clinical manifestation of the same biological disorder. Revealing the mechanism by which NPHP1 regulates the amount of INPP5E at the vertebrate TZ and elucidating the relevant signaling pathways represent important steps toward developing novel therapeutic targets.

Because its defects are associated with many severe ciliopathies, the function of $\mathrm{TZ}$ is an active field of biomedical research. Numerous publications have demonstrated that TZ proteins serve as the ciliary gatekeeper, but the exact mechanisms by which they regulate ciliary gating are still not clear. Although previous studies have shown that NPHP1 acts as a ciliary gate in C. elegans (Williams et al., 2011), NPHP1 deficiency did not alter ciliary levels of Arl13b or Sstr3 in vertebrate cells, suggesting that NPHP1 is not involved in governing the transition of these proteins in vertebrate cilia (Lin, Guo, \& Dutcher, 2018). More recent studies have proposed that the TZ could act as a specific ciliary gate for lipids (Goncalves \& Pelletier, 2017). Supporting this notion, the location of INPP5E within vertebrate cilia has been shown to depend on TZ proteins, such as TCTN1. RPGRIP1L/MKS5 has been reported to play a role in regulating the amount of the phosphoinositide PIP2 within cilia of $C$. elegans, but little is known about the possible involvement of NPHP1 as a ciliary gate for lipids. In the present study, we report a diagnosed case of Senior-Løken syndrome, which derives from a deletion of a key TZ protein. The patient had renal failure and retinal degeneration. We show that primary cilia in the patient's renal biopsy exhibit morphological abnormalities which likely impaired normal function. Importantly, we also show that the ciliary localization of INPP5E is altered in the sample from the patient with NPHP1 deletion, confirming our hypothesis that NPHP1 may act as a ciliary gate for lipids. Furthermore, we detected an abnormal expression of NPHP4, which suggests that NPHP1 acts as a structural scaffold for the NPHP4 present in the TZ. We bring into focus the input of NPHP1 in forming a TZ in which NPHP4 functions normally, which in turn regulates INPP5E transition and its ability to influence cilia stability.

\section{Figure legends}

\section{Fig. 1. Early retinal degeneration and kidney cyst in a patient with NPHP1 deletion.}

(A) Representative color fundus photographs of the patient with NPHP1 deletion showing normal fundus in both eyes without optic disc pallor or retinal dystrophy. (B) Vision field examination demonstrating bilateral visual field loss in this patient. The grayscale plots present as a cluster of paracentral points with decreased sensitivity and scotoma. (C) Full field standard ERG of OD showing increased implicit time and reduced amplitude in scotopic 0.01 ERG, and in photopic 3.0 ERG. (D) Representative hematoxylin and eosin $(\mathrm{H} \& \mathrm{E})$-stained kidney sections showing the histological pattern in normal human and the patient with NPHP1 deletion. Kidney specimen is shown at low (left) and high (right) magnification. Kidney histology of NPHP1 patient illustrates the characteristic triad of corticomedullary cysts, tubular basement membrane 
disruption, and tubulointerstitial nephropathy. Scale bars = (left) 200 $\mu \mathrm{m}$; (right) $50 \mu \mathrm{m}$. Transmission electron micrographs of primary cilia and TBM in kidney epithelial cells from the NPHP1 deletion patient. TEM reveals thickened tubular membrane and decreased cilia. Scale bars $=2 \mu \mathrm{m}$.

Fig. 2. Disorganized cilia with abnormal ciliary protein expression in renal tubular cells of patient with NPHP1 deletion.

(A) Disorganized cilia in renal tubular cells of patient with NPHP1 deletion. Confocal immunomicroscopy of normal and patient kidney sections stained with Arl13b and Ac-tubulin antibodies to detect primary cilia and with IFT 88 to detect basal body. Primary cilia appear shorter and disorganized in epithelial cells of patient kidney sections. (E- F) Quantification of ciliation and ciliary length $(\mathrm{n}=200)$. (B) Normal expression of NPHP8 and abnormal expression of (C) NPHP4 in cilia of renal tubular cells from the NPHP1 deletion patient. Confocal immunomicroscopy of patient kidney sections stained for NPHP8 and NPHP4. (G) Quantification of cells with abnormal expression of NPHP4 $(n=50)$. (D) Aberrant expression of phosphoinositide 5 -phosphatases in cilia of renal tubular cells from the NPHP1 deletion patient. Confocal immunomicroscopy of patient kidney sections stained for INPP5E. $(\mathrm{H})$ Quantification of cells with abnormal expression $(\mathrm{n}=50)$. Statistical analysis in E-H was performed using Student's test, $\mathrm{p}<0.05$ was considered statistically significant. Scale bars: (A-C) $10 \mu \mathrm{m}$; (D) magnified images $40 \mu \mathrm{m}$. (Arrow, marks primary cilia)

\section{Acknowledgments:}

We thank Dr. Tia Kowal for improving the manuscript.

\section{Data Availability Statement}

The data that support the findings of this study are available on request from the corresponding author. The data are not publicly available due to privacy or ethical restrictions.

\section{References}

Aggarwal, H. K., Jain, D., Yadav, S., Kaverappa, V., \& Gupta, A. (2013). Senior-loken syndrome with rare manifestations: a case report.Eurasian J Med, 45 (2), 128-131. doi:10.5152/eajm.2013.25

Anderson, C. T., Castillo, A. B., Brugmann, S. A., Helms, J. A., Jacobs, C. R., \& Stearns, T. (2008). Primary cilia: cellular sensors for the skeleton. Anat Rec (Hoboken), 291 (9), 1074-1078. doi:10.1002/ar.20754

Awata, J., Takada, S., Standley, C., Lechtreck, K. F., Bellve, K. D., Pazour, G. J., . . . Witman, G. B. (2014). NPHP4 controls ciliary trafficking of membrane proteins and large soluble proteins at the transition zone. J Cell Sci, 127 (Pt 21), 4714-4727. doi:10.1242/jcs.155275

Berbari, N. F., O'Connor, A. K., Haycraft, C. J., \& Yoder, B. K. (2009). The primary cilium as a complex signaling center. Curr Biol, 19 (13), R526-535. doi:10.1016/j.cub.2009.05.025

Chavez, M., Ena, S., Van Sande, J., de Kerchove d'Exaerde, A., Schurmans, S., \& Schiffmann, S. N. (2015). Modulation of Ciliary Phosphoinositide Content Regulates Trafficking and Sonic Hedgehog Signaling Output. Dev Cell, 34 (3), 338-350. doi:10.1016/j.devcel.2015.06.016

Constable, S., Long, A. B., Floyd, K. A., Schurmans, S., \& Caspary, T. (2020). The ciliary phosphatidylinositol phosphatase Inpp5e plays positive and negative regulatory roles in Shh signaling.Development, 147 (3). doi:10.1242/dev.183301

Delous, M., Hellman, N. E., Gaude, H. M., Silbermann, F., Le Bivic, A., Salomon, R., . . . Saunier, S. (2009). Nephrocystin-1 and nephrocystin-4 are required for epithelial morphogenesis and associate with PALS1/PATJ and Par6. Hum Mol Genet, 18 (24), 4711-4723. doi:10.1093/hmg/ddp434

Dyson, J. M., Conduit, S. E., Feeney, S. J., Hakim, S., DiTommaso, T., Fulcher, A. J., . . Mitchell, C. A. (2017). INPP5E regulates phosphoinositide-dependent cilia transition zone function. J Cell Biol, 216 (1), 247-263. doi:10.1083/jcb.201511055 
Fliegauf, M., Horvath, J., von Schnakenburg, C., Olbrich, H., Muller, D., Thumfart, J., . . . Omran, H. (2006). Nephrocystin specifically localizes to the transition zone of renal and respiratory cilia and photoreceptor connecting cilia. J Am Soc Nephrol, 17 (9), 2424-2433. doi:10.1681/ASN.2005121351

Garcia-Gonzalo, F. R., Corbit, K. C., Sirerol-Piquer, M. S., Ramaswami, G., Otto, E. A., Noriega, T. R., . . . Reiter, J. F. (2011). A transition zone complex regulates mammalian ciliogenesis and ciliary membrane composition. Nat Genet, 43 (8), 776-784. doi:10.1038/ng.891

Garcia-Gonzalo, F. R., \& Reiter, J. F. (2017). Open Sesame: How Transition Fibers and the Transition Zone Control Ciliary Composition. Cold Spring Harb Perspect Biol, 9 (2). doi:10.1101/cshperspect.a028134

Gogendeau, D., Lemullois, M., Le Borgne, P., Castelli, M., Aubusson-Fleury, A., Arnaiz, O., . . Tassin, A. M. (2020). MKS-NPHP module proteins control ciliary shedding at the transition zone.PLoS Biol, 18 (3), e3000640. doi:10.1371/journal.pbio.3000640

Goncalves, J., \& Pelletier, L. (2017). The Ciliary Transition Zone: Finding the Pieces and Assembling the Gate. Mol Cells, 40 (4), 243-253. doi:10.14348/molcells.2017.0054

Halbritter, J., Porath, J. D., Diaz, K. A., Braun, D. A., Kohl, S., Chaki, M., . . . Group, G. P. N. S. (2013). Identification of 99 novel mutations in a worldwide cohort of 1,056 patients with a nephronophthisis-related ciliopathy. Hum Genet, 132 (8), 865-884. doi:10.1007/s00439-013-1297-0

Hildebrandt, F., Attanasio, M., \& Otto, E. (2009). Nephronophthisis: disease mechanisms of a ciliopathy. $J$ Am Soc Nephrol, 20 (1), 23-35. doi:10.1681/ASN.2008050456

Jacoby, M., Cox, J. J., Gayral, S., Hampshire, D. J., Ayub, M., Blockmans, M., . . . Schurmans, S. (2009). INPP5E mutations cause primary cilium signaling defects, ciliary instability and ciliopathies in human and mouse. Nat Genet, 41 (9), 1027-1031. doi:10.1038/ng.427

Jiang, S. T., Chiou, Y. Y., Wang, E., Lin, H. K., Lee, S. P., Lu, H. Y., . . . Li, H. (2008). Targeted disruption of Nphp1 causes male infertility due to defects in the later steps of sperm morphogenesis in mice. Hum Mol Genet, 17 (21), 3368-3379. doi:10.1093/hmg/ddn231

Lewis, W. R., Bales, K. L., Revell, D. Z., Croyle, M. J., Engle, S. E., Song, C. J., . . . Yoder, B. K. (2019). Mks6 mutations reveal tissue- and cell type-specific roles for the cilia transition zone. FASEB J, 33 (1), 1440-1455. doi:10.1096/fj.201801149R

Lin, H., Guo, S., \& Dutcher, S. K. (2018). RPGRIP1L helps to establish the ciliary gate for entry of proteins. J Cell Sci, 131 (20). doi:10.1242/jcs.220905

Omran, H. (2010). NPHP proteins: gatekeepers of the ciliary compartment.J Cell Biol, 190 (5), 715-717. doi: $10.1083 /$ jcb.201008080

R, H. (2014). Senior- loken syndrome - a ciliopathy. J Clin Diagn Res, 8 (11), MD04-05. doi: $10.7860 / J C D R / 2014 / 9688.5120$

Ronquillo, C. C., Bernstein, P. S., \& Baehr, W. (2012). Senior-Loken syndrome: a syndromic form of retinal dystrophy associated with nephronophthisis. Vision Res, 75 , 88-97. doi:10.1016/j.visres.2012.07.003

Sang, L., Miller, J. J., Corbit, K. C., Giles, R. H., Brauer, M. J., Otto, E. A., . . . Jackson, P. K. (2011). Mapping the NPHP-JBTS-MKS protein network reveals ciliopathy disease genes and pathways. Cell, 145 (4), 513-528. doi:10.1016/j.cell.2011.04.019

Satir, P., \& Christensen, S. T. (2007). Overview of structure and function of mammalian cilia. Annu Rev Physiol, 69 , 377-400. doi:10.1146/annurev.physiol.69.040705.141236

Shi, X., Garcia, G., 3rd, Van De Weghe, J. C., McGorty, R., Pazour, G. J., Doherty, D., . . . Reiter, J. F. (2017). Super-resolution microscopy reveals that disruption of ciliary transition-zone architecture causes Joubert syndrome. Nat Cell Biol, 19 (10), 1178-1188. doi:10.1038/ncb3599 
Srivastava, S., \& Sayer, J. A. (2014). Nephronophthisis. J Pediatr Genet, 3 (2), 103-114. doi:10.3233/PGE14086

Stokman, M., Lilien, M., \& Knoers, N. (1993). Nephronophthisis. In M. P. Adam, H. H. Ardinger, R. A. Pagon, S. E. Wallace, L. J. H. Bean, K. Stephens, \& A. Amemiya (Eds.), GeneReviews ((R)) . Seattle (WA).

Szymanska, K., \& Johnson, C. A. (2012). The transition zone: an essential functional compartment of cilia. Cilia, 1 (1), 10. doi:10.1186/2046-2530-1-10

Veland, I. R., Awan, A., Pedersen, L. B., Yoder, B. K., \& Christensen, S. T. (2009). Primary cilia and signaling pathways in mammalian development, health and disease. Nephron Physiol, 111 (3), p39-53. doi:10.1159/000208212

Williams, C. L., Li, C., Kida, K., Inglis, P. N., Mohan, S., Semenec, L., . . . Leroux, M. R. (2011). MKS and NPHP modules cooperate to establish basal body/transition zone membrane associations and ciliary gate function during ciliogenesis. J Cell Biol, 192 (6), 1023-1041. doi:10.1083/jcb.201012116

Williams, C. L., Masyukova, S. V., \& Yoder, B. K. (2010). Normal ciliogenesis requires synergy between the cystic kidney disease genes MKS-3 and NPHP-4. J Am Soc Nephrol, 21 (5), 782-793. doi:10.1681/ASN.2009060597

Wolf, M. T. (2015). Nephronophthisis and related syndromes. Curr Opin Pediatr, 27 (2), 201-211. doi:10.1097/MOP.0000000000000194

Zhou, W., Otto, E. A., Cluckey, A., Airik, R., Hurd, T. W., Chaki, M., . . . Hildebrandt, F. (2012). FAN1 mutations cause karyomegalic interstitial nephritis, linking chronic kidney failure to defective DNA damage repair. Nat Genet, 44 (8), 910-915. doi:10.1038/ng.2347 
A
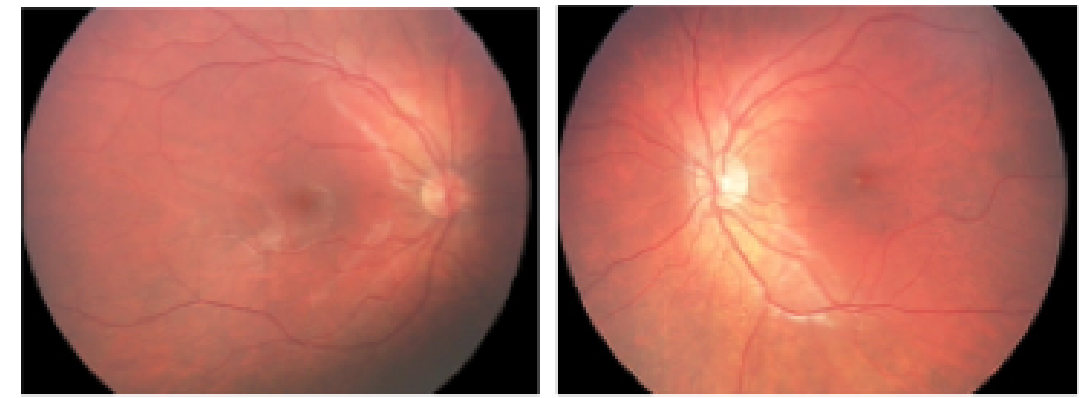

B

C
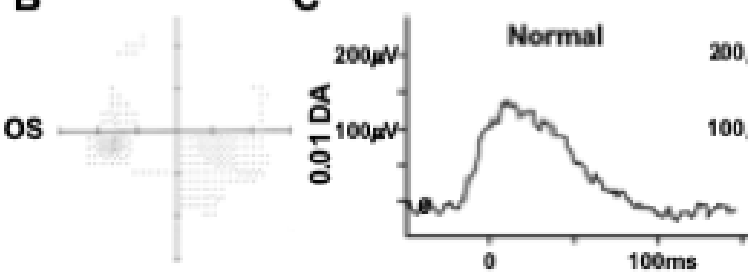

$200 \mathrm{mV}$ NPHP1 pationt
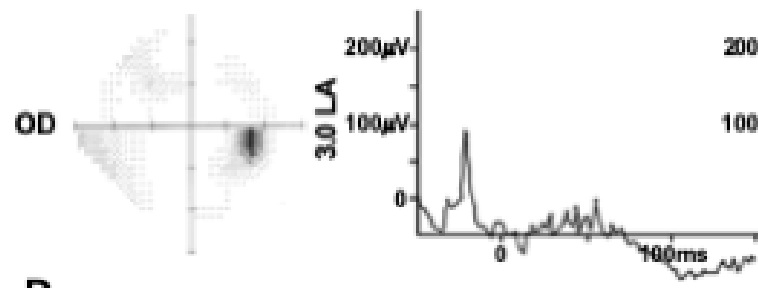

$200 \mu \mathrm{V}$

$100 \mathrm{NW}$
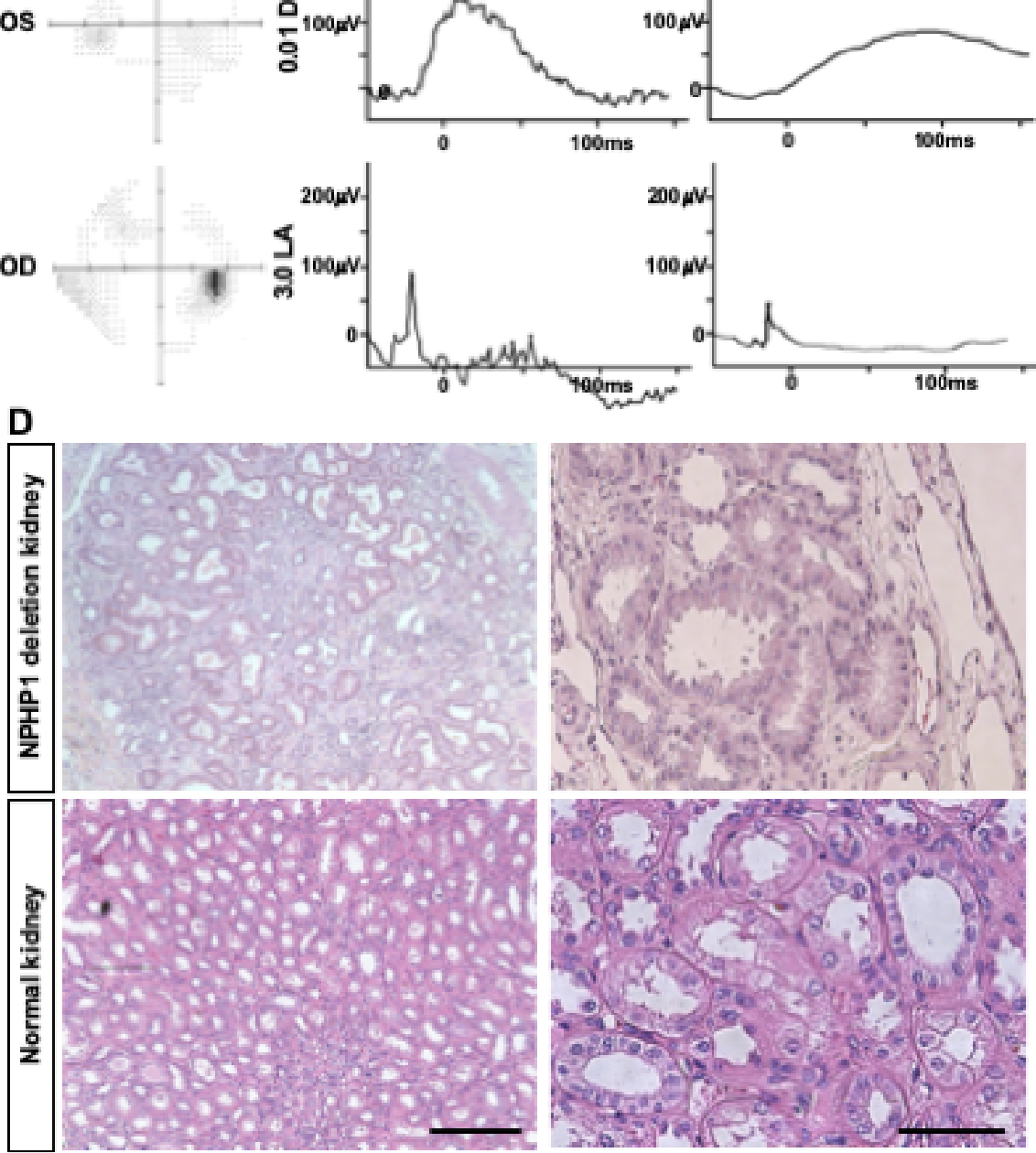

E
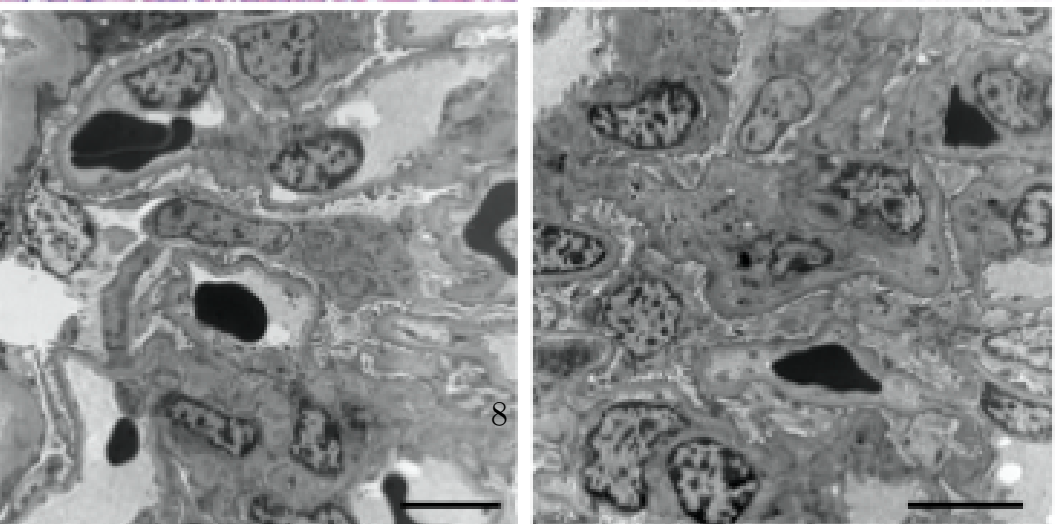

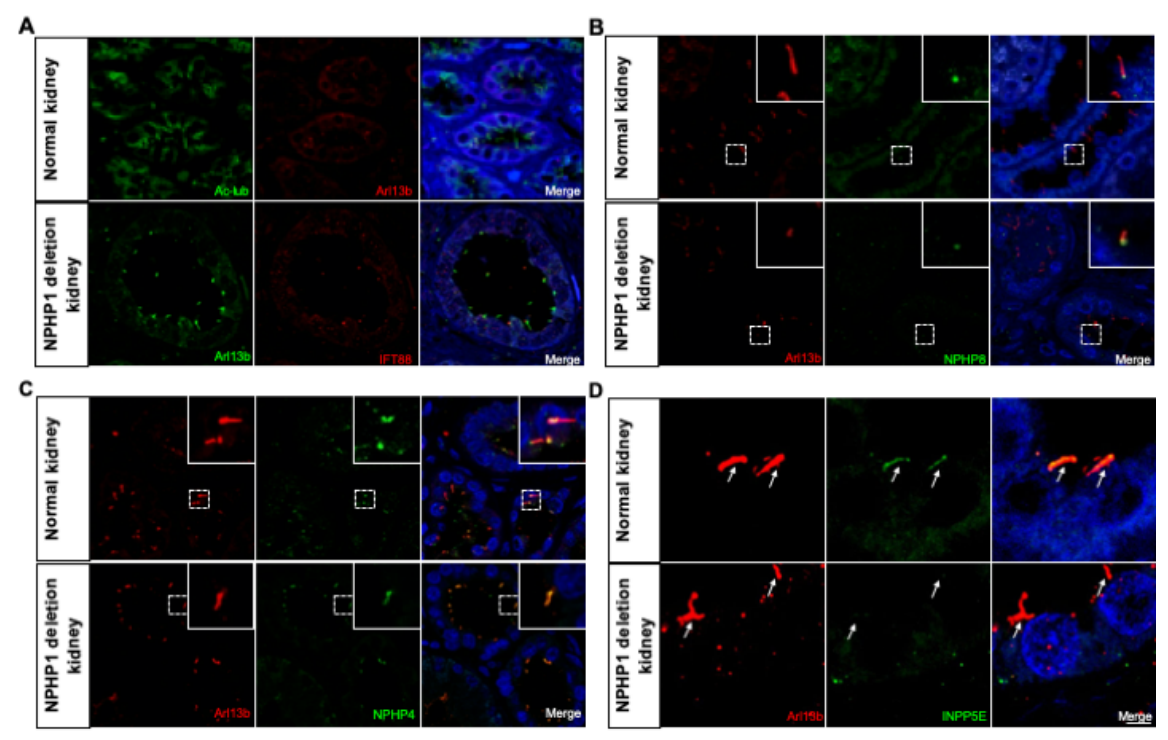

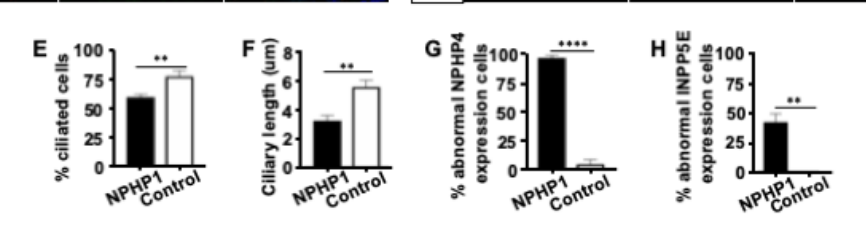

